\title{
Perfil epidemiológico dos acometidos por hanseníase em três estados da região
}

\section{Nordeste do Brasil}

\author{
Epidemiological profile of people affected by leprosy in three states in the northeast region of Brazil \\ Perfil epidemiológico de la lepra afectada en tres estados del noreste de Brasil
}

Recebido: 17/12/2021 | Revisado: 24/12/2021 | Aceito: 06/01/2022 | Publicado: 09/01/2022

Caroline Paula Marquetti

ORCID: https://orcid.org/0000-0002-5765-7025 Universidade Luterana do Brasil,Brasil

E-mail: caroline_paula@ hotmail.com

Jussara Alves Pinheiro Sommer

ORCID https://orcid.org/0000-0002-6485-4828 Universidade Luterana do Brasil, Brasil

E-mail: jussara.sommer@ulbra.br

Eliane Fraga da Silveira

ORCID: https://orcid.org/0000-0002-0992-5136 Universidade Luterana do Brasil, Brasil E-mail: eliane.silveira@ulbra.br

Nádia Teresinha Schröder

ORCID: https://orcid.org/0000-0001-5505-1137 Universidade Luterana do Brasil, Brasil E-mail: nadia.schroder@ulbra.br

Eduardo Périco

ORCID: https://orcid.org/0000-0002-2926-6246 Universidade do Vale do Taquari, Brasil E-mail:perico@univates.br

\begin{abstract}
Resumo
Objetivo: Descrever o perfil epidemiológico dos acometidos por hanseníase na Região Nordeste do Brasil. Método: Estudo ecológico, descritivo, retrospectivo, construído a partir da análise de dados secundários sobre a hanseníase registrados na Região Nordeste, no período de 2013 a 2017. Os dados foram obtidos do Sistema Informatizado de Dados das Notificações de Hanseníase (DATASUS) e do Sistema de Informação de Agravos de Notificação (SINAN). A partir dos dados, foi calculada a frequência absoluta, a taxa de incidência, a prevalência e a taxa de incremento anual (TIA) $(\mathrm{p} \leq$ 0,05). Resultados: Foram diagnosticados 180.019 casos novos de hanseníase no Brasil, e a Região Nordeste apresentou 77.669 novos casos, representando 43,13\% do total. Em relação ao perfil dos indivíduos acometidos, a maioria é do sexo masculino e com faixa etária entre 30 e 49 anos. A forma virchowiana foi a prevalente no Maranhão $(55,8 \%)$; e a dimorfa, na Bahia (34,7\%) e em Pernambuco (34,3\%). Analisando a TIA, nos estados da Região Nordeste, observa-se estabilidade das taxas de prevalência em Pernambuco e na Bahia e declínio no Maranhão. Conclusão: Os três estados analisados não atingiram as metas para redução de detecção de novos casos nem redução nos casos de Grau II. As variáveis sociodemográficas dos portadores apontam a manutenção de desigualdades socioeconômicas que impactam na qualidade de vida dos portadores. Essa investigação poderá contribuir com o desenvolvimento de políticas públicas para a promoção da saúde da população afetada. Salienta-se a importância de outros estudos que aprofundem a investigação no Maranhão, que se mantém com números elevados da doença.

Palavras-chave: Mycobacterium leprae; Região nordeste; Epidemiologia; Vulnerabilidade social.
\end{abstract}

\begin{abstract}
Aim: To describe the epidemiological profile of people affected by leprosy in the Northeast region of Brazil. Methods: Ecological, descriptive, retrospective study, resulting from the analysis of secondary data on leprosy recorded in the Northeast region in the period from 2013 to 2017. Data were obtained from the Computerized Data System of Leprosy Notifications (DATASUS) and Information System of Grievances Notification (SINAN). The absolute frequency, incidence rate, prevalence and Annual Increment Rate (TIA) were calculated ( $\mathrm{p} \leq 0.05$ ). Results: During the analyzed period, 180,019 new leprosy cases were diagnosed in Brazil, and the Northeast region showed 77,669 new cases, representing $43.13 \%$ of the total. Regarding the profile of affected individuals, most are male and aged between 30 and 49 years. The Virchowian form was more prevalent in Maranhão (55.8\%) and Dimorpha in Bahia (34.7\%) and Pernambuco (34.3\%). Regarding the TIA, in the states of the Northeast region, there is stability in the prevalence rates for Pernambuco and Bahia, and a decrease in Maranhão. Conclusion: The three analyzed states did not meet the targets for new cases reduction, as well as reduction in Degree II cases. The sociodemographic variables of patients indicate the prevalence of socioeconomic inequalities that impact patient's life quality. This investigation may contribute to the development of public policies to improve health conditions of the affected
\end{abstract}


population and highlights the need of further studies that deepen the investigation in Maranhão, which shows the high rates for this disease.

Keywords: Mycobacterium leprae; Northeast region; Epidemiology; Social vulnerability.

\begin{abstract}
Resumen
Objetivo: Describir el perfil epidemiológico de los afectados por la enfermedad de Hansen en la región Noreste de Brasil. Método: Estudio ecológico, descriptivo, retrospectivo, construido a partir del análisis de datos secundarios sobre la enfermedad de Hansen registrados en la región Noreste de 2013 a 2017. Los datos se obtuvieron del Departamento de Informática del Sistema Único de Salud (DATASUS) y del Sistema de Información de Enfermedades de Notificación Obligatoria (SINAN). A partir de los datos, se calculó la frecuencia absoluta, la tasa de incidencia, la prevalencia y tasa de incremento anual (TIA) $(\mathrm{p} \leq 0,05)$. Resultados: En el período analizado, se diagnosticaron 180.019 nuevos casos de la enfermedad de Hansen en Brasil, y la región Noreste presentó 77.669 nuevos casos, lo que representa el $43,13 \%$ del total. En cuanto al perfil de los individuos afectados, la mayoría son varones y tienen entre 30 y 49 años. La forma Virchowiana fue más prevalente en Maranhão $(55,8 \%)$ y Dimorfa en Bahía $(34,7 \%)$ y Pernambuco $(34,3 \%)$. Analizando el TIA, en los estados de la región Noreste, hay estabilidad en las tasas de prevalencia en Pernambuco y Bahía y descenso en Maranhão. Conclusión: Los tres estados analizados no cumplieron con las metas de reducción en la detección de nuevos casos y reducción en casos de grado II. Esta investigación puede contribuir al desarrollo de políticas públicas para la promoción de la salud de la población afectada, destacando la importancia de otros estudios que profundizan la investigación en Maranhão, que mantienen altos números de la enfermedad.
\end{abstract}

Palabras clave: Mycobacterium leprae; Región noreste; Epidemiología; Vulnerabilidad social.

\title{
1. Introdução
}

A hanseníase é classificada como uma Doença Tropical Negligenciada (DTN) e sua ocorrência, geralmente, está associada às más condições socioeconômicas da população (OMS, 2021). É uma doença infectocontagiosa crônica que tem como agente etiológico o Mycobacterium leprae. Afeta, principalmente, nervos periféricos, pele e olhos (Brasil, 2020). Ocasiona sintomas dermatoneurológicos, com alterações sensitivas e motoras (Brasil, 2017); apresenta elevado poder incapacitante, podendo ocasionar deformidades físicas e até a invalidez quando não tratada adequadamente (Melo et al., 2019).

Estudos indicam que o contágio é uma combinação de vários fatores, entre eles os socioambientais, a carga parasitária e a suscetibilidade genética do indivíduo (Maymone et al., 2020; Fava et al., 2020). Pesquisas sobre hanseníase relacionando as condições humanas e o número de casos apontam que a incidência é maior nos segmentos mais pobres da população e, portanto, mais vulneráveis, já que suas condições socioeconômicas são desfavoráveis, o que facilita a contaminação e a propagação do agente etiológico (Lopes \& Rangel, 2014, OMS, 2016, Pinheiro et al., 2017; Van Brakel et al., 2012).

Houve um avanço no tratamento da hanseníase em escala mundial (OMS, 2021) por meio do Programa Estratégia Global para Hanseníase com objetivos e metas quinquenais. As metas do Programa nos períodos de 2005-2010 e 2011-2016 se concentraram no acesso ao tratamento (poliquimioterapia) e na eliminação da hanseníase como problema de saúde pública, definida como menos de um caso em tratamento por 10.000 habitantes (OMS, 2016). A estratégia para o período 2021-2030, chamada de "Rumo a zero hanseníase", propõe novas estratégias que se concentram na interrupção da transmissão e na obtenção de zero caso autóctone (OMS, 2021).

O diagnóstico é baseado no número e no tipo de lesões à pele, sendo classificada em duas formas: paucibacilar (PB), com até cinco lesões e com baciloscopia de raspado intradérmico negativo; e multibacilar (MB), com seis ou mais lesões de pele ou baciloscopia positiva. Nesta classe pode ocorrer a hanseníase virchowiana (HV) com lesões como máculas, pápulas e/ou nódulos eritematosos, podendo apresentar infiltração difusa e espessamento da pele (Scollard et al., 2020). Quanto à forma clínica, a hanseníase paucibacilar é classificada como indeterminada e tuberculoide; e a multibacilar, em dimorfa e virchowiana; já o grau de incapacidade é definido em Grau I e Grau II (Ministério da Saúde, 2017).

O Brasil aderiu aos compromissos internacionais para eliminar o agravo, entretanto não alcançou as metas de eliminação da hanseníase propostas na Estratégia 2016-2020. A doença ainda é considerada um problema de saúde pública no Brasil (Pinheiro et al., 2021 \& Brasil, 2021), com notificação compulsória e investigação obrigatória em todo o território 
nacional (Brasil, 2017).

A hanseníase possui distribuição geográfica heterogênea, em todo o território brasileiro. As regiões Sul e Sudeste apresentam os menores números de novos casos, mantendo-se com média endemicidade. As regiões Norte, Centro-Oeste e Nordeste apresentam as maiores taxas de endemicidade e de prevalência (Brasil, 2021). O Brasil é classificado como um país de alta carga para a doença e ocupa o segundo lugar na relação de países com maior número de casos no mundo, atrás apenas da Índia no ranking mundial (Gracie et al., 2017; OMS, 2019; Blok et al., 2020).

O estudo teve por objetivo conhecer o perfil epidemiológico dos casos de hanseníase na macrorregião Nordeste e a análise do número de casos nos estados do Maranhão, Pernambuco e Bahia. Diante do grave problema de saúde pública a pesquisa se justifica por sua relevância em descrever as prevalências e as características clínicas do agravo, contribuindo para elaboração de políticas e de ações no enfrentamento da hanseníase voltadas para populações específicas.

\section{Metodologia}

Trata-se de um estudo ecológico, descritivo, retrospectivo (Pereira et al., 2018; Estrela, 2018, Koche,2011), constituído a partir da análise de dados secundários sobre a hanseníase nos Estados da Região Nordeste, entre 2013 a 2017. Os dados epidemiológicos foram obtidos do banco do Departamento de Informática do Sistema Único de Saúde do Ministério da Saúde (DATASUS), utilizando o banco de dados do Sistema de Notificação de Informações de Agravo (SINAN). Informações sobre o tamanho populacional foram obtidas do Instituto Brasileiro de Geografia e Estatística (IBGE), a partir do Censo de 2010 (Instituto Brasileiro de Economia e Estatística [IBGE], 2010). As variáveis analisadas no período estipulado foram sexo, idade, escolaridade e as condições epidemiológicas da hanseníase (grau, classificação e forma) e o tamanho da população.

A Região Nordeste possui população estimada em 57.374.243 milhões de habitantes, correspondendo a um terço da população do País (IBGE, 2020). Possui área territorial de $1.558 .000 \mathrm{~km}^{2}$, é constituída por nove estados: Alagoas, Bahia, Ceará, Maranhão, Paraíba, Pernambuco, Piauí, Rio Grande do Norte e Sergipe. A análise foi realizada com os dados epidemiológicos dos estados da Bahia, Pernambuco e Maranhão em função do elevado número de casos novos diagnosticados no período analisado.

Para a classificação de endemicidade da hanseníase, foi utilizado o coeficiente de prevalência, que foi calculado utilizando o número de casos da doença dividido pela população residente estimada para o sexo, a faixa etária, as características da doença e o ano, multiplicado por 10.000 mil habitantes. O coeficiente de prevalência é classificado como baixo (0,00 a 0,99/10.000 habitantes), médio (de 1,00 a 4,99/10.000 habitantes), alto (5,00 a 9,99/10.000 habitantes), muito alto (10,00 a 19,99/10.000 habitantes) e hiper endêmico (acima de 20,00/10.000 habitantes) (Ribeiro, Silva \& Oliveira, 2018).

Nas análises estatísticas, foi utilizada a regressão de Prais-Winsten para entender a dinâmica da doença por variável no período analisado. A partir desse método foi possível entender se a doença apresentou tendências de crescimento, diminuição ou estabilidade durante o período e nos diferentes estados analisados. O cálculo baseou-se no estudo de Antunes (2005) e aplicado por Böhm et al. (2016), que calculou a Taxa de Incremento Anual (TIA) a partir dos dados da regressão: TIA $=[-1+(10 b)]^{*} 100$, em que $b$ é o coeficiente de inclinação da reta na regressão Prais-Winsten. As tendências foram consideradas significativas quando os valores de $p$ da regressão apresentaram $\alpha \leq 0,05$. Caso contrário, as tendências foram consideradas estáveis. Essa análise foi elaborada no programa estatístico R Project 4.03.

A pesquisa foi realizada com dados secundários, de domínio público, sem identificação dos sujeitos, portanto obedecendo aos princípios éticos da Resolução 196/96 do Conselho Nacional de Saúde, o que justifica a ausência do parecer do Comitê de Ética em Pesquisa (Brasil, 2012).

\section{Resultados}


No período analisado foram registradas 134.794 mil pessoas com hanseníase no território brasileiro; as regiões Nordeste (58.363) e Norte (26.330) apresentaram os maiores números de casos, correspondendo a 43,3\% e a 19,5\% dos casos de hanseníase no período de cinco anos (Tabela 1). Nesse período, em 2014, houve o maior número de casos registrados (31.061), seguido pelo ano de 2015 (28.761).

Tabela 1: Número de casos registrados por região do Brasil, no período de 2013 a 2017.

\begin{tabular}{lccccccc}
\hline \multicolumn{1}{c}{ Período } & Norte & Nordeste & Sudeste & Sul & Centro-Oeste & Ign & Total \\
\hline 2013 & 5445 & 11193 & 4271 & 1086 & 4694 & 125 & 26814 \\
2014 & 6068 & 13420 & 4484 & 1021 & 5852 & 216 & 31061 \\
2015 & 5147 & 12760 & 4013 & 1.013 & 5623 & 205 & 28761 \\
2016 & 5053 & 10898 & 3571 & 826 & 4666 & 204 & 25218 \\
2017 & 4617 & 10092 & 2738 & 716 & 4653 & 124 & 22940 \\
\hline Total & 26330 & 58363 & 19077 & 4662 & 25488 & 874 & 134794 \\
\hline
\end{tabular}

Fonte: SINAN.

A Região Nordeste apresentou entre as cinco regiões analisadas do País o maior número de casos registrados no SINAN no período analisado. Considerando os estados de Pernambuco, Maranhão e Bahia, um total de 51.752 novos infectados com hanseníase foram registrados no sistema. No período de 2013 a 2015 ocorreu o maior número de notificações entre os três estados, e o Maranhão apresentou o maior número de registros (14.791) (Tabela 2).

Tabela 2: Número de casos de pessoas com hanseníase registrados no SINAN, nos estados de Pernambuco, Maranhão e Bahia, no período de 2013 a 2017.

\begin{tabular}{lccccccccccc}
\hline \multirow{2}{*}{ Estados } & \multicolumn{2}{c}{2013} & \multicolumn{2}{c}{2014} & \multicolumn{2}{c}{2015} & \multicolumn{2}{c}{2016} & \multicolumn{2}{c}{2017} & Total \\
\cline { 2 - 9 } & \multicolumn{2}{c}{$\mathrm{n}(\%)$} & \multicolumn{2}{c}{$\mathrm{n}(\%)$} & $\mathrm{n}(\%)$ & $\mathrm{n}(\%)$ & $\mathrm{n}(\%)$ & & \\
\hline Pernambuco & 3181 & $(29,5)$ & 3147 & $(28,9)$ & 2856 & $(27,4)$ & 2424 & $(26,3)$ & 3183 & $(30,5)$ & 14791 \\
Maranhão & 4831 & $(44,9)$ & 4538 & $(41,7)$ & 4481 & $(43,0)$ & 4233 & $(45,9)$ & 4403 & $(42,2)$ & 22486 \\
Bahia & 2754 & $(25,6)$ & 3210 & $(29,5)$ & 3082 & $(29,6)$ & 2570 & $(27,9)$ & 2859 & $(27,4)$ & 14475 \\
\hline Total & $10766(100)$ & $10895(100)$ & $10419(100)$ & $9227(100)$ & $10445(100)$ & 51752 \\
\hline
\end{tabular}

Fonte: SINAN.

Analisando a TIA, observa-se estabilidade das taxas de prevalência nos estados de Pernambuco e Bahia, entretanto o Estado do Maranhão apresenta declínio, com decréscimo de 0,62\% ao ano (Tabela 3).

Tabela 3: Situação da prevalência de hanseníase nos estados analisados entre 2013-2017.

\begin{tabular}{lccc}
\hline \multicolumn{1}{c}{ Estado de Notificação } & Taxa de Incremento Anual (TIA) & $p$ valor* & Situação \\
\hline Pernambuco & -0.068 & 0,863 & Estável \\
Bahia & 0.003 & 0,997 & Estável \\
Maranhão & -0.620 & $\mathbf{0 , 0 1 6}$ & Declínio \\
\hline
\end{tabular}

*Valores em negrito alfa $<0,05$. Fonte: Autores (2021).

O coeficiente de prevalência da hanseníase nos estados de Pernambuco, Maranhão e Bahia ficou acima do coeficiente do País no período analisado. Os coeficientes de prevalência no Brasil e na Região Nordeste, conforme a classificação, foram 
considerados como médio (1,00 a 4,99/10.000 hab.) (Figura 1). Maranhão e Pernambuco estão com os coeficientes superiores aos encontrados para a Região Nordeste. O Maranhão é classificado como alto (5,00 a 9,99/10.000 hab.); e Pernambuco e Bahia são classificados como médios (1,00 a 4,99/10.000 hab.).

Figura 1: Coeficiente de prevalência de hanseníase nos estados, na Região Nordeste e no Brasil, entre 2013 e 2015.

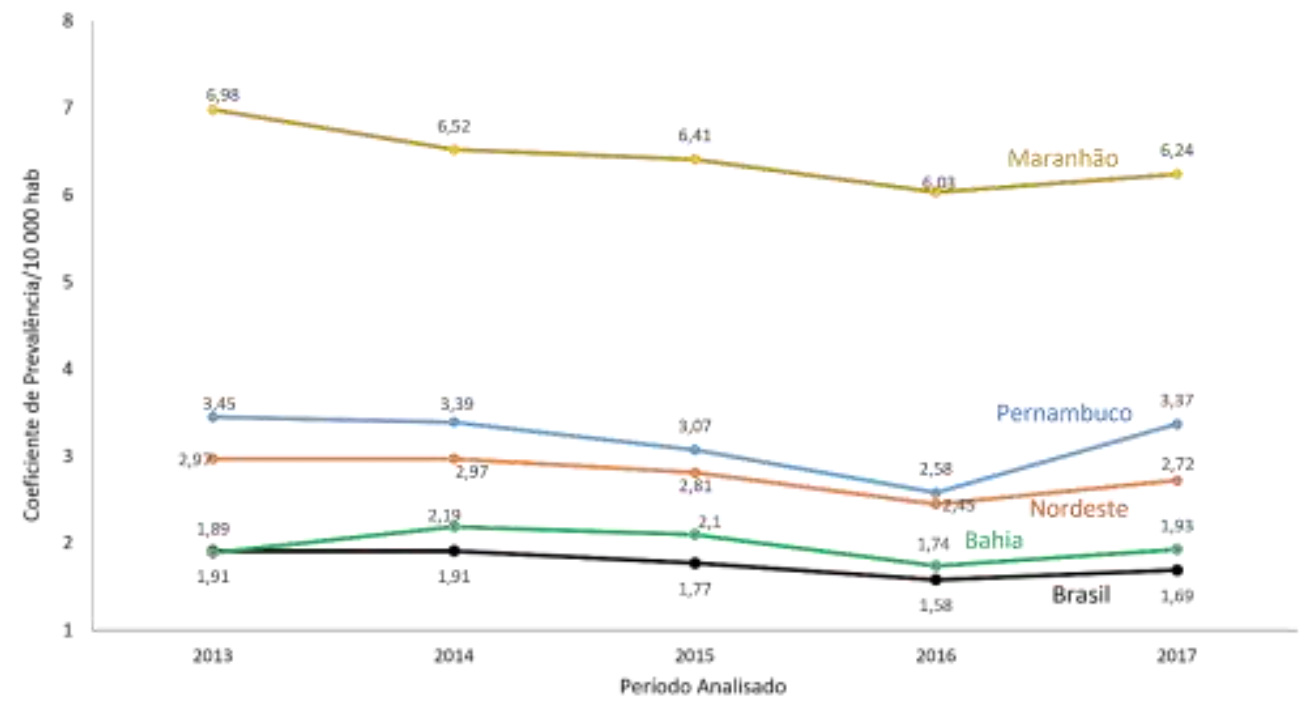

Fonte: Autores (2021).

Em relação ao perfil dos indivíduos notificados no sistema SINAN, a maioria é do sexo masculino. No Maranhão os homens atingem 57,7\% dos casos; na Bahia e em Pernambuco estão próximos dessa proporção. Os indivíduos pardos são os mais notificados em todos os estados analisados, com maior percentual no Maranhão (66,7\%). O tempo de estudo e o número de indivíduos com hanseníase nos estados foram semelhantes, entretanto os percentuais mais elevados ocorreram em indivíduos com o Ensino Fundamental incompleto. Uma informação relevante é sobre a incompletude dos dados no sistema SINAN em relação à Bahia $(23,1 \%)$ e a Pernambuco $(29,1 \%)$, correspondendo ao total de 15.284 pessoas sem informação sobre sua escolaridade (Tabela 4). 
Tabela 4. Perfil dos indivíduos com hanseníase nos Estados do Maranhão, Bahia e Pernambuco, no período de 2013 a 2017.

\begin{tabular}{|c|c|c|c|c|c|c|c|}
\hline & \multirow{2}{*}{ Variável } & \multicolumn{2}{|c|}{ Maranhão } & \multicolumn{2}{|c|}{ Bahia } & \multicolumn{2}{|c|}{ Pernambuco } \\
\hline & & $\mathrm{n}$ & $(\%)$ & $\mathrm{n}$ & $(\%)$ & $\mathrm{n}$ & $(\%)$ \\
\hline \multirow{3}{*}{ Sexo } & Ignorado & 2 & $(0,0)$ & 2 & $(0,0)$ & 2 & $(0,0)$ \\
\hline & Masculino & 25966 & $(57,7)$ & 15198 & $(52,5)$ & 15032 & $(50,8)$ \\
\hline & Feminino & 19004 & $(42,3)$ & 13750 & $(47,5)$ & 14548 & $(49,2)$ \\
\hline \multirow{12}{*}{ Escolaridade } & Ign/Branco & 4688 & $(10,4)$ & 6674 & $(23,1)$ & 8610 & $(29,1)$ \\
\hline & Analfabeto & 7044 & $(15,7)$ & 2524 & $(8,7)$ & 2440 & $(8,2)$ \\
\hline & 1. ${ }^{\mathrm{a}}$ a $4 .^{\mathrm{a}}$ série Inc. EF & 10132 & $(22,5)$ & 6308 & $(21,8)$ & 5362 & $(18,1)$ \\
\hline & 4. ${ }^{\mathrm{a}}$ série Comp. EF & 3014 & $(6,7)$ & 1794 & $(6,2)$ & 2126 & $(7,2)$ \\
\hline & 5. ${ }^{\mathrm{a}}$ a $8 .^{\mathrm{a}}$ série Inc. EF & 6938 & $(15,4)$ & 3874 & $(13,4)$ & 3986 & $(13,5)$ \\
\hline & Ensino F. Completo & 2622 & $(5,8)$ & 1326 & $(4,6)$ & 1252 & $(4,2)$ \\
\hline & Ensino Médio Inc. & 2876 & $(6,4)$ & 1498 & $(5,2)$ & 1520 & $(5,1)$ \\
\hline & Ensino M. Completo & 5840 & $(13,0)$ & 3626 & $(12,5)$ & 2938 & $(9,9)$ \\
\hline & Educação Superior Inc. & 498 & $(1,1)$ & 314 & $(1,1)$ & 258 & $(0,9)$ \\
\hline & Educação S. Completa & 856 & $(1,9)$ & 756 & $(2,6)$ & 596 & $(2,0)$ \\
\hline & Não se aplica & 464 & $(1,0)$ & 256 & $(0,9)$ & 494 & $(1,7)$ \\
\hline & Ign/Branco & 682 & $(1,5)$ & 1578 & $(5,5)$ & 2430 & $(8,2)$ \\
\hline \multirow{4}{*}{ Raça } & Branca & 6276 & $(14,0)$ & 4116 & $(14,2)$ & 5534 & $(18,7)$ \\
\hline & Preta & 7404 & $(16,5)$ & 4692 & $(16,2)$ & 3886 & $(13,1)$ \\
\hline & Amarela & 464 & $(1,0)$ & 196 & $(0,7)$ & 222 & $(0,8)$ \\
\hline & Parda & 29986 & $(66,7)$ & 18250 & $(63,0)$ & 17420 & $(58,9)$ \\
\hline \multirow{12}{*}{$\begin{array}{l}\text { Faixa Etária } \\
\quad \text { (anos) }\end{array}$} & Indígena & 160 & $(0,4)$ & 118 & $(0,4)$ & 90 & $(0,3)$ \\
\hline & 1 a 4 & 130 & $(0,3)$ & 94 & $(0,3)$ & 168 & $(0,6)$ \\
\hline & 5 a 9 & 1328 & $(3,0)$ & 604 & $(2,1)$ & 982 & $(3,3)$ \\
\hline & 10 a 14 & 2580 & $(5,7)$ & 1232 & $(4,3)$ & 1520 & $(5,1)$ \\
\hline & 15 a 19 & 2590 & $(5,8)$ & 1322 & $(4,6)$ & 1584 & $(5,4)$ \\
\hline & 20 a 29 & 6380 & $(14,2)$ & 3190 & $(11,0)$ & 3388 & $(11,5)$ \\
\hline & 30 a 39 & 8160 & $(18,1)$ & 5372 & $(18,6)$ & 5450 & $(18,4)$ \\
\hline & 40 a 49 & 7010 & $(15,6)$ & 5330 & $(18,4)$ & 5432 & $(18,4)$ \\
\hline & 50 a 59 & 6976 & $(15,5)$ & 5184 & $(17,9)$ & 5076 & $(17,2)$ \\
\hline & 60 a 69 & 5482 & $(12,2)$ & 3820 & $(13,2)$ & 3618 & $(12,2)$ \\
\hline & 70 a 79 & 3196 & $(7,1)$ & 1990 & $(6,9)$ & 1808 & $(6,1)$ \\
\hline & $80+$ & 1140 & $(2,5)$ & 812 & $(2,8)$ & 556 & $(1,9)$ \\
\hline
\end{tabular}

Fonte: SINAN.

A faixa etária entre 30 e 49 anos apresentou maior número de indivíduos notificados nos três estados analisados. No Maranhão o maior percentual (18,1\%) ocorreu entre 30 e 39 anos; e na Bahia e em Pernambuco, na faixa de 30 até 49 anos (Tabela 4). Comparando a TIA entre os sexos, todos os estados mantiveram-se estáveis ao longo dos anos, de forma que o Maranhão apresentou declínio no sexo masculino, com taxa de 0,64\% ao ano (Tabela 5). 
Tabela 5. Análise da Taxa de Incremento Anual (TIA) entre os sexos, nos Estados de Pernambuco, Bahia e Maranhão, no período entre 2013 e 2017.

\begin{tabular}{lcccc}
\hline \multirow{2}{*}{ Estado de Notificação } & Sexo & $\begin{array}{c}\text { Taxa de Incremento Anual } \\
\text { (TIA) }\end{array}$ & $p$ valor* & Situação \\
\hline \multirow{2}{*}{ Pernambuco } & Feminino & -0.0986 & 0,805 & Estável \\
& Masculino & -0.0365 & 0,921 & Estável \\
\multirow{2}{*}{ Bahia } & Feminino & -0.1412 & 0,867 & Estável \\
& Masculino & 0.0869 & 0,896 & Estável \\
Maranhão & Feminino & -0.3841 & 0,227 & Estável \\
& Masculino & -0.6445 & $\mathbf{0 , 0 0 3 0 4}$ & Declínio \\
\hline
\end{tabular}

*Valores em negrito $\alpha \leq 0,05$. Fonte: Autores (2021).

Em relação à idade, em Pernambuco, as faixas etárias de cinco a nove e de 20 a 29 anos apresentaram declínio no período; e as demais tenderam à estabilidade. Na Bahia, em declínio somente entre 20 e 29 anos; as demais faixas etárias tenderam à estabilidade. No Maranhão, as faixas etárias de 20 a 29; 30 a 39 e 60 a 69 anos apresentaram declínio (Tabela 6).

Tabela 6. Análise da Taxa de Incremento Anual (TIA) por faixa etária nos Estados de Pernambuco, Bahia e Maranhão.

\begin{tabular}{|c|c|c|c|c|c|c|c|c|c|}
\hline \multirow{2}{*}{$\begin{array}{l}\text { Faixa etária } \\
\quad(\text { anos })\end{array}$} & \multicolumn{3}{|c|}{ Pernambuco } & \multicolumn{3}{|c|}{ Bahia } & \multicolumn{3}{|c|}{ Maranhão } \\
\hline & TIA & p-valor* & Situação & TIA & $p$ valor* & Situação & TIA & p-valor* & Situação \\
\hline 1 a 4 & $-0,897$ & 0,304 & Estável & $-0,23$ & 0,953 & Estável & 0,931 & 0,769 & Estável \\
\hline 5 a 9 & $-0,916$ & 0,004 & Declínio & 0,383 & 0,765 & Estável & 0 & 0,999 & Estável \\
\hline 10 a 14 & 0,001 & 0,979 & Estável & $-0,246$ & 0,668 & Estável & $-0,014$ & 0,974 & Estável \\
\hline 15 a 19 & $-0,197$ & 0,8 & Estável & $-0,096$ & 0,935 & Estável & $-0,123$ & 0,848 & Estável \\
\hline 20 a 29 & $-0,62$ & 0,048 & Declínio & $-0,904$ & 0,05 & Declínio & $-0,35$ & 0,009 & Declínio \\
\hline 30 a 39 & $-0,127$ & 0,673 & Estável & $-0,598$ & 0,146 & Estável & $-0,543$ & 0,005 & Declínio \\
\hline 40 a 49 & 0,041 & 0,872 & Estável & 0,242 & 0,823 & Estável & $-0,218$ & 0,303 & Estável \\
\hline 50 a 59 & $-0,006$ & 0,297 & Estável & $-0,012$ & 0,975 & Estável & 0 & 0,941 & Estável \\
\hline 60 a 69 & 0,037 & 0,813 & Estável & 0 & 0,951 & Estável & $-0,353$ & $\mathbf{0 , 0 3 6}$ & Declínio \\
\hline 70 a 79 & $-0,003$ & 0,476 & Estável & $-0,009$ & 0,288 & Estável & $-0,147$ & 0,081 & Estável \\
\hline $80 \mathrm{e}+$ & 0,018 & 0,937 & Estável & 0,054 & 0,843 & Estável & 0,022 & 0,821 & Estável \\
\hline
\end{tabular}

*Valores em negrito $\alpha \leq 0.05$. Fonte: Autores (2021).

Em relação à forma clínica, classe operacional e grau de incapacidade, observa-se que a forma virchowiana foi a prevalente no Maranhão (55,8\%); a forma dimorfa foi a prevalente na Bahia $(34,7 \%)$ e em Pernambuco $(34,3 \%)$. Na classe operacional, a multibacilar foi a prevalente nos três estados. O Maranhão se destacou com 77,1\% dos indivíduos notificados. No grau de incapacidade, os maiores percentuais estão classificados no Grau I, com amplitude de 70,3\% a 75,8\% (Figura 2). 
Figura 2. Percentual de indivíduos registrados de acordo com as características clínicas do agravo nos Estados do Maranhão, Bahia e Pernambuco, no período entre 2013 e 2017.

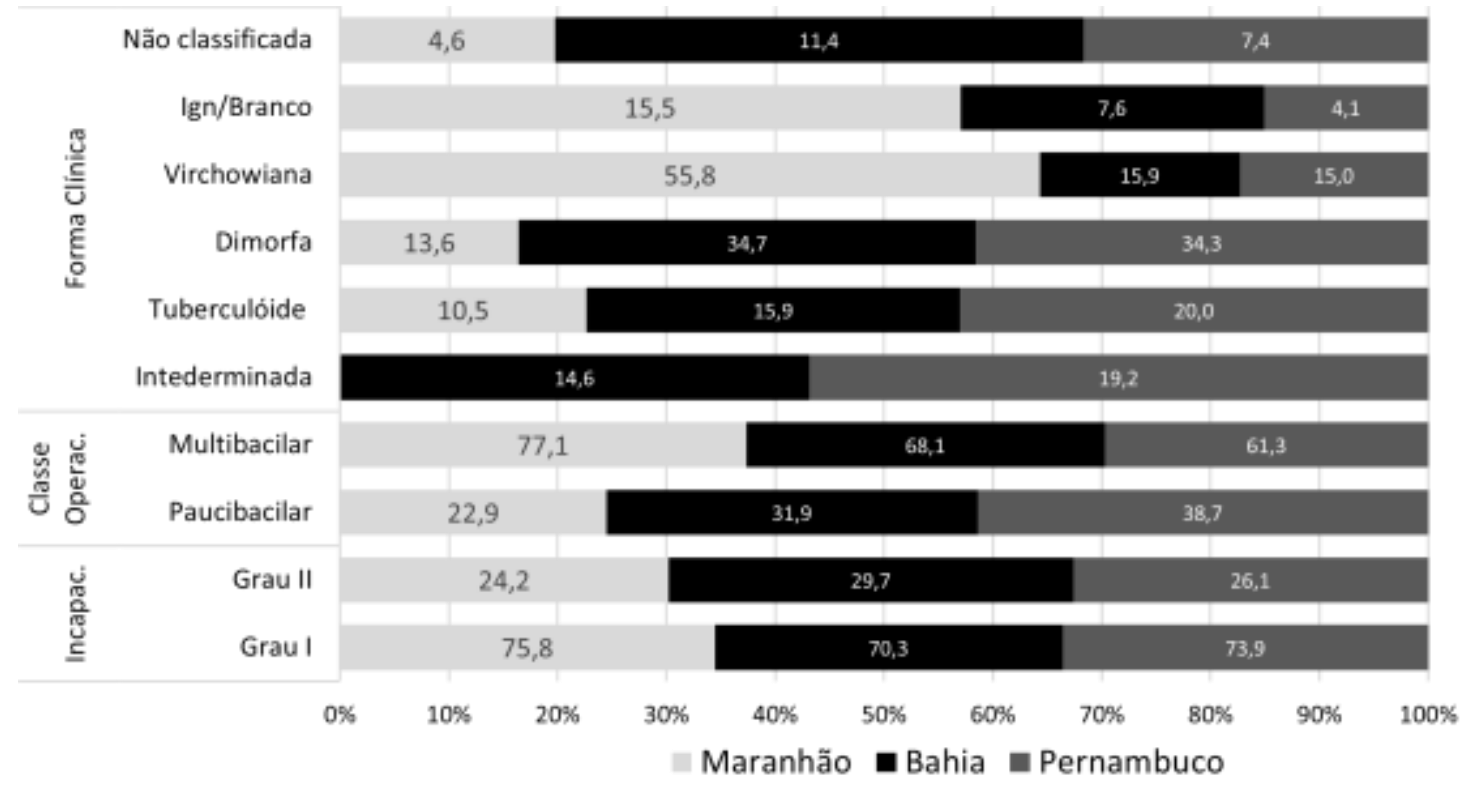

Fonte: Autores (2021).

$\mathrm{Na}$ análise estatística, quanto à TIA em relação à classe operacional, a prevalência da classe multibacilar foi estável ao longo dos anos. Entretanto a classe paucibacilar apresentou declínio nos três estados (Tabela 7).

Tabela 7. Análise da Taxa de Incremento Anual (TIA) por classe operacional diagnosticada de hanseníase nos Estados de Pernambuco, Bahia e Maranhão.

\begin{tabular}{ccccc}
\hline \multirow{2}{*}{ Estado de notificação } & $\begin{array}{c}\text { Classe } \\
\text { Operac. Diag. }\end{array}$ & $\begin{array}{c}\text { Taxa de Incremento Anual } \\
\text { TIA }\end{array}$ & $\boldsymbol{p}$ valor* & Situação \\
\hline \multirow{2}{*}{ Pernambuco } & Paucibacilar & $-0,760$ & $\mathbf{0 , 0 0 0}$ & Declínio \\
& Multibacilar & $-0,970$ & 0,619 & Estável \\
\multirow{2}{*}{ Bahia } & Paucibacilar & $-0,654$ & $\mathbf{0 , 0 5 8}$ & Declínio \\
& Multibacilar & 0,272 & 0,759 & Estável \\
\multirow{2}{*}{ Maranhão } & Paucibacilar & 0,428 & $\mathbf{0 , 0 0 8}$ & Declínio \\
& Multibacilar & $-0,223$ & 0,796 & Estável \\
\hline
\end{tabular}

*Valores em negrito apresentam alfa <0,05. Fonte: Autores (2021).

Em relação à forma clínica, a dimorfa foi estável ao longo dos anos. Entretanto a forma tuberculoide apresentou declínio nos três estados; e a virchowiana, no Maranhão, também apresentou declínio (Tabela 8). 
Tabela 8. Análise da Taxa de Incremento Anual (TIA) em relação à forma clínica notificada no SINAN, nos Estados de Pernambuco, Bahia e Maranhão.

\begin{tabular}{|c|c|c|c|c|}
\hline Estado de notificação & Forma Clínica & $\begin{array}{c}\text { Taxa de Incremento Anual } \\
\text { TIA }\end{array}$ & p-valor* & Situação \\
\hline \multirow{6}{*}{ Pernambuco } & Ign/Branco & 11,13 & 0,494 & Estável \\
\hline & Indeterminada & $-0,974$ & 0,009 & Declínio \\
\hline & Tuberculoide & $-0,936$ & 0,001 & Declínio \\
\hline & Dimorfa & 0,507 & 0,678 & Estável \\
\hline & Virchowiana & $-0,224$ & 0,918 & Estável \\
\hline & Não classificada & 4,661 & 0,612 & Estável \\
\hline \multirow{6}{*}{ Bahia } & Ign/Branco & $-1,000$ & 0,058 & Estável \\
\hline & Indeterminada & $-1,000$ & 0,132 & Estável \\
\hline & Tuberculoide & $-0,997$ & 0,012 & Declínio \\
\hline & Dimorfa & 0,737 & 0,686 & Estável \\
\hline & Virchowiana & $-0,614$ & 0,866 & Estável \\
\hline & Não classificada & 24,882 & 0,524 & Estável \\
\hline \multirow{6}{*}{ Maranhão } & Ign/Branco & - & & \\
\hline & Indeterminada & $-0,930$ & 0,012 & Declínio \\
\hline & Tuberculoide & $-0,906$ & 0,001 & Declínio \\
\hline & Dimorfa & 0,337 & 0,810 & Estável \\
\hline & Virchowiana & $-0,972$ & 0,015 & Declínio \\
\hline & Não classificada & $-0,975$ & 0,458 & Estável \\
\hline
\end{tabular}

*Valores em negrito apresentam $\alpha \leq 0,05$. Fonte: Autores (2021).

Em relação ao grau de incapacidade, os Estados de Pernambuco e Bahia apresentaram as prevalências entre as duas modalidades de incapacidade (I, II) na situação de estável ao longo dos anos. Entretanto, no Maranhão, o Grau II apresentou declínio (Tabela 9).

Tabela 9. Análise da Taxa de Incremento Anual (TIA) em relação ao grau de incapacidade notificado no SINAN, nos Estados de Pernambuco, Bahia e Maranhão, no período analisado.

\begin{tabular}{ccccc}
\hline \multirow{2}{*}{ Estado de notificação } & Aval. Incap. & TIA & p-valor* & Situação \\
\hline \multirow{2}{*}{ Pernambuco } & Grau I & 0,4259 & 0,81 & Estável \\
& Grau II & 25,0615 & 0,56 & Estável \\
\multirow{2}{*}{ Bahia } & Grau I & $-0,9996$ & 0,07 & Estável \\
& Grau II & $-1,0000$ & 0,11 & Estável \\
\multirow{2}{*}{ Maranhão } & Grau I & 0,9329 & 0,71 & Estável \\
& Grau II & $-0,9997$ & $\mathbf{0 , 0 0}$ & Declínio \\
\hline
\end{tabular}

*Valores em negrito apresentam $\alpha \leq 0,05$. Fonte: Autores (2021).

\section{Discussão}

A Região Nordeste registrou o maior número geral de casos notificados de hanseníase no período analisado. O Estado do Maranhão apresentou coeficiente de prevalência classificada como alta, entretanto apresentou declínio, enquanto Pernambuco e Bahia apresentaram elevação. Essa tendência foi registrada por Ribeiro et al. (2018), que identificaram redução na taxa de prevalência de hanseníase no Brasil, no ano 2015, com 1,01/10.000 habitantes. Entretanto a taxa voltou a aumentar no ano de 2018, com 1,48/10.000 habitantes, permanecendo no parâmetro médio (Boletim Epidemiológico, 2020). 
Os homens são os mais afetados em todas as faixas etárias e em escolaridade com até quatro anos de estudos, mas se observa elevado percentual de analfabetos no Maranhão. Em relação ao número de notificação com informação ignorado/branco, é elevado nos três estados. Esses dados são similares aos divulgados nos Boletins Epidemiológicos para Hanseníase entre 2018 e 2020 (Brasil, 2018, Brasil, 2019, Brasil, 2020). Estudos indicam que há evidências de uma associação significativa do nível educacional com o grau de incapacidade física na Índia (Guthi, Arepalli \& Ganapa, 2016) e no Brasil (Ribeiro, 2015).

Em 2018, a taxa de detecção geral de casos novos no Brasil foi classificada como de alta endemicidade, e a taxa de prevalência permaneceu no parâmetro "médio" (Ministério da Saúde, 2020). A redução da prevalência de casos de hanseníase não foi homogênea entre as regiões do País; as maiores taxas foram registradas no Nordeste, no Norte e no Centro-Oeste. Na Região Nordeste, o estado com mais alta prevalência é o Maranhão (Ribeiro et al., 2018). Esses dados coincidem com os observados neste estudo.

Os indivíduos autodeclarados pardos e pretos, nos três estados investigados, são mais acometidos. Esse dado é coerente com a distribuição demográfica da população por cor/raça no País (IBGE, 2010). Os altos percentuais de notificados com baixa escolaridade entre aqueles autodeclarados pardos e pretos têm relação com os fatores socioeconômicos e podem ser relevantes na ocorrência da hanseníase, a qual em diferentes contextos espaciais qualifica distintas especificidades e vulnerabilidades das populações; já a detecção de casos é diretamente associada aos municípios com maior percentual de pobreza e desigualdade social, entretanto o estudo realizado em Tocantins, entre 2002 e 2012, identificou relação entre o acesso ao Programa Bolsa Família e a melhoria na qualidade de vida (renda familiar, redução da insegurança alimentar, melhora das condições nutricionais, aumento do acesso e frequência escolar), que apresentaram significativamente menor risco para hanseníase (Rosa et al., 2017).

O Ministério da Saúde realizou análise de todas as regiões do Brasil, de sorte que a Região Nordeste teve a maior proporção de analfabetos (Brasil, 2020). Estudo envolvendo a escolaridade como fator de aumento nas incapacidades por hanseníase, realizado no Vale do Jequitinhonha-MG, revelou associação significativa entre o grau de incapacidade e o nível de escolaridade, com predominância de indivíduos com até quatro anos de estudo; e a análise estatística mostrou uma correlação inversamente proporcional entre escolaridade e grau de incapacidade (Lages, Kerr, Bueno, Niitsuma \& Lana, 2018).

Neste estudo, a forma indeterminada teve declínio no Maranhão e em Pernambuco. O Maranhão apresentou as maiores taxas de incapacidade de Grau I, classe operacional multibacilar e forma clínica virchowiana. Apesar do declínio verificado nas taxas, os dados apontam o Maranhão como estado com alta carga da doença, contaminação ativa e diagnóstico tardio. Esses dados são corroborados em pesquisa realizada com 43 portadores de hanseníase em Recife (D’Azevedo et al., 2021). A presença de incapacidades físicas é considerada um indicador do nível de conhecimento sobre as manifestações clínicas da hanseníase, do acesso aos serviços de saúde e da habilidade das equipes da Atenção Primária à Saúde no diagnóstico e no acompanhamento dos casos (OMS, 2016).

\section{Considerações Finais}

Apesar da redução de casos de hanseníase nos últimos anos, no Brasil, as taxas de detecção e endemicidade são elevadas nas macrorregiões Centro-Oeste, Norte e Nordeste. Na Região Nordeste se destacam os Estados do Maranhão, Bahia e Pernambuco, que, após serem analisados, não atingiram as metas previstas para redução na detecção de novos casos gerais nem redução nos casos de Grau II, conforme previsto na Estratégia Global para Hanseníase 2016-2020 (OMS, 2020).

Essa é uma doença que prevalece em condições de pobreza, contribuindo na manutenção do quadro de desigualdade, já que esses fatores representam um travamento no desenvolvimento dos países. As variáveis sociodemográficas dos portadores apontam a continuidade de desigualdades socioeconômicas que impactam na qualidade de vida dos portadores. 
Dessa forma, é fundamental o planejamento e o desenvolvimento de políticas públicas que visem prevenir a doença, diminuir o número de casos, estimular o diagnóstico precoce e, além disso, criar projetos de educação em saúde de forma a auxiliar na diminuição do preconceito contra os portadores da doença. Portanto, ações de esclarecimento e atenção continuada podem auxiliar na redução das taxas de abandono do tratamento, ampliando o acesso ao diagnóstico e melhorando a qualidade de atendimento. Esta investigação poderá contribuir com o desenvolvimento de políticas públicas que apontem para a promoção da saúde da população afetada e a divulgação de mais informações sobre a doença, de forma a possibilitar a redução do preconceito em relação aos portadores.

Indica-se a necessidade de outros estudos que aprofundem investigação e analise de dados sociodemograficos, no Estado do Maranhão visando entender o motivo pelo qual os números da hanseniase se mantêm elevados quando comparados aos outros estados da mesma região.

\section{Referências}

Antunes, J. L. F. (2005). Mortalidade por câncer e desigualdade social em São Paulo. [tese]. Universidade de São Paulo.

Barbosa, D. R. M., Almeida, M. G. \& Santos, A. G. (2014). Perfil epidemiológico da hanseníase em cidade hiperendêmica do Maranhão. Revista Rede de Cuidados em Saúde, 8(1), 01-13.

Böhm, A. W., Costa, C. S., Neves, R. G., Flores, T. R. F. \& Nunes, B. P. (2016). Tendência da incidência de dengue no Brasil, 2002-2012. Epidemiologia e Serviços de Saúde, 25(4), 725-733.

Blok D. J., Vlas, S. J. De \& Richardus, J. H. (2015). Global elimination of leprosy by 2020: are we on track? Parasites \& Vectors, 8(1).

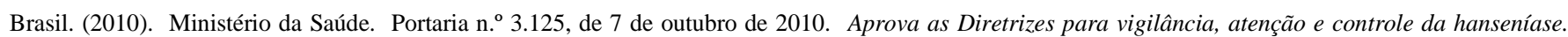
Diário Oficial da União, Brasília (DF), 2010 out 15; Seção 1:55. http://revista.iec.gov.br/submit/index.php/rpas/article/view/389/298

Brasil. (2012). Ministério da Saúde. Conselho Nacional de Saúde. Resolução $n .^{\circ} 466$, de 12 de dezembro de 2012 . Aprova normas regulamentadoras de pesquisas envolvendo seres humanos. Brasília (DF): Ministério da Saúde. https://bvsms.saude.gov.br/bvs/saudelegis/ cns/2013/res0466_12_12_2012.html

Brasil. (2016) Ministério da Saúde. Secretaria de Vigilância em Saúde. Departamento de Vigilância das Doenças Transmissíveis. Diretrizes para vigilância, atenção e eliminação da hanseníase como problema de saúde pública. Brasília: Ministério da Saúde. http://www.aids.gov.br/pt-br/pub/2020/boletimepidemiologico-de-hanseniase-2020 .

Brasil. (2017). Ministério da Saúde. .Portaria de consolidação n. ${ }^{\circ} 4$, de 28 de setembro de 2017. Consolidação das normas sobre os sistemas e os subsistemas do Sistema Único de Saúde.

Brasil. (2018). Ministério da Saúde, Secretaria de Vigilância em Saúde. Boletim Epidemiológico. 4(49).

Brasil (2019). Ministério da Saúde. Boletim Epidemiológico de Hanseníase/2019.

Brasil. (2020). Ministério da Saúde. Secretaria de Vigilância em Saúde. Ministério da Saúde. Número Especial. http://www.aids.gov.br/pt-br/pub/2020/boletim-epidemiologico-de-hanseniase-2020.

Costa, N. M. G. B., Barbosa, T. C. S., Queiroz, D. T., Oliveira, A. K. A., Montemezzo, L. C. D. \&, Andrade, U. C. (2020). Perfil sociodemográfico e grau de incapacidade do portador de hanseníase em um centro de referência no Estado do Ceará. Braz J Develop. 6(6), 41439-49.

D’Azevedo, S. S. P., Santos, D. C. M., Arruda, G. A., Barbosa, J. C., Alves, M. G. T. \&, Souza, N. M. N. (2021). Profile of functionality of persons affected by leprosy. Rev Rene. 22:e61702.

Estrela, C. (2018). Metodologia Científica: Ciência, Ensino, Pesquisa. Editora Artes Médicas.

Fava V. M., et al. (2020). Genetics of leprosy: today and beyond. Human Genetics. 139(6), 835-846.

Gracie, R. et al. (2017). Análise da distribuição geográfica dos casos de hanseníase. Rio de Janeiro, 2001 a 2012. Ciência e Saúde Coletiva.. 22(5).

Guthi, V. R., Arepalli, S. \& Ganapa, P. (2016). Study of socio demographic factors among persons affected by leprosy in Kurnool division of Kurnool district, Andhra Pradesh, India. International Journal of Community Medicine and Public Health, 3(12), 3548- 3555.

IBGE. (2012). Censo Demográfico 2010. Resultados do Universo agregado por setores e Censo 2000 e 2010 . Resultados do Universo agregado por setores. Censo Brasileiro de 2010. IBGE. https://www.ibge.gov.br/estatisticas/sociais/saude/9662-censo-demografico-2010.html?=\&t=o-que-e

IBGE. (2021). Instituto Brasileiro de Geografia e Estatística. Cidades. https://cidades.ibge.gov.br/

Lages, D. S., Kerr, B. M., Bueno, I. C., Niitsuma, E. N. A. \& Lana, F. C. F.. (2018). A baixa escolaridade está associada ao aumento de incapacidades físicas no diagnóstico de hanseníase no Vale do Jequitinhonha. HU Revista Juiz de Fora, 44(3), 303-309.

Lopes, V. A. S. \& Rangel, E. M. (2014). Hanseníase e vulnerabilidade social: uma análise do perfil socioeconômico de usuários em tratamento irregular. 
Research, Society and Development, v. 11, n. 1, e38811124872, 2022

(CC BY 4.0) | ISSN 2525-3409 | DOI: http://dx.doi.org/10.33448/rsd-v11i1.24872

Saúde em Debate. 38(103), 817-829.

Maymone M, et al. (2020). Leprosy: Clinical aspects and diagnostic techniques. J Am Acad Derma. 83(1), 1-14.

Monteiro, L. D., Monteiro, R.M. S. M., Martins-Melo, F. R., Alencar, C. H. A. \&, Heukelbach, J.. Determinantes sociais da hanseníase em um estado hiperendêmico da Região Norte do Brasil. Rev Saúde Pública. 2017;51:70 https://doi.org/10.1590/S1518-8787.2017051006655

Melo, J . P., Moraes, M. M., Santos, N. R. \& Santos, T. S. (2017). Perfil epidemiológico dos casos de hanseníase de uma unidade de saúde. Revista de Saúde Coletiva da UEFS, 7(1), 29-34.

Monken, M., Peiter, P., Barcellos, C., Rojas, L. I., Navarro, M. B. M. A., Gondim, G. M., \& Gracie, R. (2008). O território na saúde: construindo referências para análises em saúde e ambiente. Miranda AC, Barcellos C, Moreira JC, Monken M, organizadores. Território, ambiente e saúde. Fiocruz, 23-41.

OMS (2016). Organização Mundial da Saúde. Estratégia Global para Hanseníase 2016-2020: aceleração rumo a um mundo sem hanseníase. Nova Delhi: Biblioteca da Organização Mundial da Saúde. https://apps.who.int/iris/bitstream/ha ndle/10665/208824/9789290225201-pt.pdf?sequence=17

OMS (2021). Organização Mundial da Saúde. Estratégia Global de Hanseníase 2021-2030. "Rumo a zero hanseníase”. Nova Delhi: Organização Mundial da Saúde, Escritório Regional para o Sudeste Asiático. Licence: CC BY-NCSA 3.0 IGO. http://apps.who.int/iris

Pereira, A. S. et al. (2018).Metodologia da Pesquisa Científica. UFSM.

Pinheiro, M. G. C., Miranda, F. A. N, Simpson, C. A, Carvalho, F. P. B., Ataide, C. A. V. \& Lira, A. L. B. C. (2017). Compreendendo a "alta em hanseníase": uma análise de conceito. Revista Gaúcha de Enferm.. 38(4), 1-8.

Ribeiro, M. D. A., Silva, J. C. A. \& Oliveira, S. B. (2018). Estudo epidemiológico da hanseníase no Brasil: reflexão sobre as metas de eliminação. Rev Panam. Salud Pública. 42:e 42.

Ribeiro, G. C. \& Lana, F. C. F. (2015). Incapacidades físicas em hanseníase: Caracterização, fatores relacionados e evolução. Cogitare Enfermagem, 20(3), 496-503.

Van Brakel, W. H, Sihombing, . B, Djarir, H., Beise, K., Kusumawardhani, L. \& Yulihane R et al. (2012). Disability in people affected by leprosy: the role of impairment, activity, social participation, stigma and discrimination. Glob Health Action. (5),.1-11. 